\title{
Wallace, Turner, and Perkins revisited
}

\author{
R. J. SENTER, DAVID O. RICHTER, SANDRA D. WILSON, \\ and DEBBIE CLEMENTS \\ University of Cincinnati, Cincinnati, Ohio 45221
}

\begin{abstract}
A quasi-replication of the often cited 1957 Wallace, Turner, and Perkins study failed to reproduce the striking performance of $99 \%$ and $95 \%$ recall of 500 and 700 PA nouns, after one "mental imagery" learning trial, which was reported in the original study. One subject in the present study was, however, found to exhibit correct recall of $75.8 \%$ of 348 PA response members and $87.5 \%$ of 154 pairs involving two concrete nouns, while under instructions to use a mental imagery learning strategy.
\end{abstract}

In 1957, Wallace, Turner, and Perkins published a rather remarkable set of findings familiar to, and often cited by, researchers in the field of imaginal mnemonics. In their study, Wallace et al. reported that subjects previously naive to the use of mental images were able to perform a paired associate (PA) memory task involving 500 and 700 pairs with, respectively, $99 \%$ and $95 \%$ correct recall after only a single exposure to the pairs. Wallace et al. discounted the possibility that their subjects were individuals possessing exceptional memory capacities by citing mediocre performance shown by the same subjects on routine memory tasks performed outside their main experimental procedure. The rather phenomenal performance reported was attributed to the fact that the subjects were instructed to use associative mental images as their memory mediators. Due to the facts that this study has been so often cited as being rather substantial evidence of the power of associative mental imagery as a memory enhancer and that the subjects' performances reported were so remarkable, a replication of the study has been warranted for some time. To our knowledge, no such replication has been published. This lack of replication has, no doubt, been largely due to the obvious difficulty of getting experimental subjects to volunteer for such a grueling task, involving the memorization of hundreds of PA nouns in a single sitting. After several years of attempted recruiting, we were finally able to induce two undergraduate students to agree to attempt the task. The present paper is, then, a report of our attempt to perform a quasireplication of the Wallace et al. experiment.

\section{METHOD}

\section{Subjects}

Two female undergraduates at the University of Cincinnati, S.W. and J.S. valiantly volunteered to be subjects. Neither participant had any experience with the use of imaginal mnemonics prior to the beginnings of the present study.

Requests for reprints should be sent to R. J. Senter, Department of Psychology, Mail Location 376, University of Cincinnati, Cincinnati, Ohio 45221 .

\section{Materials}

It would have been preferable to use the list of pairs originally employed by Wallace et al. (1957) but only 24 of their pairs were published in the original report. Personal communications with one of the authors a few years ago indicated that the complete original list of pairs was no longer available. We, therefore, created a new list by selecting, haphazardly, a few more than 1,400 nouns from a dictionary and unsystematically forming 700 pairs with these nouns. The only deliberate deviation from random pairing occurred when the two nouns had some obvious natural association (e.g., hair-brush or night-gown). When such a pairing turned up, the nouns were returned to the pool to be re-paired with less obvious partners.

\section{Procedure}

Both subjects reported to the laboratory at 9:00 a.m. on a Saturday. The experimenter explained the nature of the task, verified that the subjects were familiar with all of the individual nouns, instructed the subjects in the use of associative mental images, and provided practice examples for about $30 \mathrm{~min}$. Then the experiment began with one experimenter reading pairs of nouns to each subject alternately via earphones connected to an intercom system. The speed of presentation was self-paced by each subject (approximately $1.3 \mathrm{~min} /$ pair). The duration of the session was approximately $7.5 \mathrm{~h}$ for each subject. J.S. received 345 word pairs and S.W. received 348 word pairs during the session.

\section{RESULTS}

The most obvious results were that (1) as in the original study, the subjects asked for, and received, frequent rest periods (about $3 \mathrm{~h}$ of the $7.5 \mathrm{~h}$ were spent in rest), (2) the subjects complained a great deal, and (3) neither subject completed the hoped-for minimum of 500 pairs.

As soon as the subjects reported that they did not care to participate further, they were tested for recall by the experimenter's reading the stimulus (first) member of each pair, taking the pairs in the order in which they had been heard, and asking the subjects to write the other noun as the response member. Responses were counted as being correct only if the exact word originally given was recalled.

J.S. correctly recalled 88 of the response members, for a correct recall hit rate of $25.5 \%$. S.W. correctly 
recalled 264 of her total of 348 response members, for a hit rate of $75.8 \%$.

A second scoring was done, counting only the pairs in which both members were concrete nouns and, hence, were pairs presumably more amenable to the creation of associative mental images. In the case of J.S. 174 pairs contained two concrete nouns. Of these, she correctly recalled 56 response members $(32.2 \%)$. S.W. received 176 pairs in which both members were concrete nouns. Of these, she correctly recalled 154 of the response members $(87.5 \%)$.

The rather demanding learning task required in this study unfortunately obviated the ready availability of willing control subjects. Even though the Wallace et al. (1957) study itself included no control group, we could not, in good conscience, conclude the present study without getting some idea as to how subjects uninstructed in the mental image strategy would perform on our PA task. Toward this end, we recruited 41 introductory psychology students, who were naive in the use of mental imagery mnemonics, to participate in an exercise for course credit. To these subjects we gave a mimeographed sheet containing the first 100 PA nouns we had given to J.S. and S.W. These subjects were instructed to study each pair as long as they wished via any study method they might choose. They were instructed not to go back and restudy any pair once they had advanced to a new pair (this instruction did not, of course, preclude their restudying if they so chose). The subjects were to continue their study until they felt that they had learned the pairs as well as they could or for $45 \mathrm{~min}$, whichever came first. When each subject had finished studying, he/she was provided an answer sheet containing the first member of each pair and a blank. The subjects were given as much time as they wished to provide the proper response members.

The mean number of correct response members recorded was $40.07(S D=17.97)$. If we take the liberty of extrapolating this performance using a straight-line function to a set of 346 (the approximate mean number of PAs given to J.S. and S.W.), we estimate that subjects in our comparison group would have provided the correct response member to 139 items. If we make another intuitive plunge and assume that the variance of the distribution of correct responses based on a total set of 346 pairs would only double, with respect to that observed with 100 items, then the projected distribution of responses to 346 items would have a standard deviation of 25.41. On a distribution having these values of central tendency and variability, J.S.'s performance (88 correct responses) would have an associated $\mathrm{z}$ score of $-2.01 ;$ S.W.'s performance ( 264 correct responses) would have an associated $\mathrm{z}$ value of 4.92 . In short, J.S. scored at a level below our estimated mean for non-imagery-instructed subjects at a point roughly corresponding to the fifth centile, whereas S.W.'s score would place her performance well above the 99th centile.

\section{DISCUSSION}

The present study can, at best, be considered to be a replication of the Wallace et al. (1957) study in form, but scarcely in substance. Its only virtue is that, to our knowledge, there has been no other attempt at replication published at all. In the present study, we could not use the original PA materials. Our subjects were both female, whereas the subjects in the original study were male. These material and subject differences, among possibly countless others, could conceivably account for the discrepancies between the results of the present study and those originally reported by Wallace et al.

Unfortunately, for all the intense efforts of our subject volunteers, we have not produced data that either clearly support or refute the findings of Wallace et al. (1957). It is clear, however, that the treatment embodied in the simple admonition "form a mental image connecting each pair and remember these pairs using associative mental images" and brief practice in doing so does not uniformly, or reliably, produce the striking recall performance reported by Wallace et al. in naive subjects exposed to a large number of PA nouns.

\section{REFERENCE}

Wallace, W. H., Turner, S. H., \& Perkins, C. C. Preliminary studies of human information storage (Signal Corps Project No. 1320c). Philadephia: University of Pennsylvania, Institute for Cooperative Research, 1957.

(Received for publication July 30, 1982.) 